\title{
Unclassifiable B-cell lymphoma with features intermediate between diffuse large B-cell lymphoma and classical Hodgkin lymphoma in an AIDS patient. Report of a case and literature review
}

\author{
Valerga $M^{1,2}$, Corti $M^{1,2}$, Bruni $G^{1,2}$, Maiolo $E^{1,2}$, Campitelli $A^{3}$, Narbaitz $M^{4}$ and Metrebian $F^{4}$ \\ ${ }^{1}$ Division of HIV/AIDS, Infectious Diseases F. J. Muñiz Hospital, Buenos Aires, Argentina \\ ${ }^{2}$ Medicine Department, Infectious Diseases Orientation, University of Buenos Aires, School of Medicine, Buenos Aires, Argentina \\ ${ }^{3}$ Histopathology Laboratory, F. J. Muñiz Hospital, Buenos Aires, Argentina \\ ${ }^{4}$ National Academy of Medicine, Histopathology Laboratory, Buenos Aires, Argentina
}

\begin{abstract}
B-cell lymphoma, unclassifiable, with features intermediate between diffuse large B-cell lymphoma (DLBCL) and classic Hodgkin lymphoma (cHL) is a B-celltype of lymphoma that demonstrates clinical, morphological, and/or immunophenotypic features between cHL and DLBCL. These lymphomas are most commonly associated with mediastinal disease, but some cases have been reported in peripheral lymph node as the primary site. This category was designed as a way to include those borderline cases that cannot be classified into a single distinct disease. Typically, these cases have features intermediate between DLBCL and cHL, or include characteristics of both lymphomas. Generally, immunophenotype showed CD20 weak or variably positive, CD15 expressed, CD30+, CD45+, CD79a+, OCT2 positive, BOB1 expressed and PAX5 positive expressed in atypical lymphoid cells. Here we present a female patient with AIDS who developed an unclassified lymphoma, with features between DLBCL and cHL.
\end{abstract}

\section{Introduction}

Patients infected with human immunodeficiency virus (HIV) have a high risk to develop non-Hodgkin (NHL) lymphomas. This risk is 100 to 300 times higher in compared with the general population [1]. High active antiretroviral therapy (HAART) reduce the incidence of some subtypes of NHL, especially, primary central nervous system lymphoma (PCNSL), but the influence on other subtypes of lymphoma, including diffuse large B cell lymphoma (DLBCL), Burkitt's lymphoma (BL), plasmablastic lymphoma (PBL) and Hodgkin's disease (HD) is uncertain [2].

The named "gray zone lymphoma" include a group of aggressive B-cell lymphoma that cannot classified in a specific diagnostic category $[3,4]$. "Gray zone lymphomas" include two variants: first, those patients with features intermediate between primary mediastinal B-cell lymphoma and classical Hodgkin lymphoma (HL) and second, those that show findings of DLBCL and BL [5]. In the type-1, histopathology show hypercelullarity with diffuse or nodular pattern and ReedSternberg or similar cells [6]. These are a less frequent lymphomas, resistant to conventional chemotherapy regimens and with a poor prognosis [7].

Herein, we present a female patient with diagnosis of advanced HIV/AIDS disease, who developed a nodal lymphoproliferative disease that was diagnosed as unclassified lymphoma with intermediate histopathological characteristics between DLBCL and cHL.

\section{Case report}

A-35-year-old female, infected with the human immunodeficiency virus (HIV), was referred to the Infectious Diseases F. J. Muñiz Hospital of Buenos Aires, because she presented a one month history of fever, night sweats and weight loss $(10 \mathrm{k})$. Years ago she was started on HAART based on zidovudine, lamivudine and nevirapine with poor adherence. On examination, she was conscious, oriented in time and place. Relevant physical findings included oral candidiasis, a large adenopathic conglomerate located on the left supraclavicular region, painless, with a hard consistency with adhesion to the surface and deep planes. Abdominal examination showed bloating, diffuse pain and hepatosplenomegaly. Significant laboratory findings include hematocrit $24 \%$; WBC $9600 / \mathrm{mm}^{3}$, (78\% of neutrophils), platelets 376 $000 / \mathrm{mm}^{3}$, erythrocyte sedimentation rate $121 \mathrm{~mm}^{\text {st }} \mathrm{h}$, renal and liver functions were normal. Serological tests for HBV, HCV, VDRL and Chagas disease were negative. Epstein-Barr virus serology was positive for IgG antibodies. Lactate deshydrogenase level was of $1920 \mathrm{UI} / \mathrm{L}$; the CD4-T_cell count was 136 cell/ $\mu \mathrm{L}(8 \%)$ and the HIV plasma viral load 166000 copies/mL $\left(\log _{10} 5.2\right)$. A computed tomography scan of thorax,

${ }^{\star}$ Correspondence to: Marcelo Corti, Department of HIV/AIDS, Infectious Diseases F. J. Muñiz Hospital, Puán 381, 2nd Floor, C 1406 CQG, Buenos Aires, Argentina, E-mail: marcelocorti@fibertel.com.ar

Received: May 25, 2020; Accepted: August 04, 2020; Published: August 07, 2020 
Valerga M (2020) Unclassifiable B-cell lymphoma with features intermediate between diffuse large B-cell lymphoma and classical Hodgkin lymphoma in an AIDS patient. Report of a case and literature review

abdomen and pelvis showed large and multiple lymphadenopathy located in cervical, supraclavicular, mediastinal and retroperitoneum. Homogeneous hepatosplenomegaly was also described (Figure 1). Histopathological diagnosis was made by the left supraclavicular surgical biopsy. Biopsy smears showed a diffuse and dense proliferation of atypical lymphoid cells, with areas of necrosis and the presence of Reed-Sternberg-like cells, mononuclear cells and eosinophils (Figure 2). Therefore, immunohistochemical (IHC) techniques was performed to rule out the type of lymphoma. IHC findings revealed that neoplastic cells were positive for PAX5 (Figure 3), OCT2, BCL2 and CD79a, with a less expression of BOB-1. The atypical lymphoid cells were also positive for CD30 and MUM-1 (Figure 4) and negative for CD45 (Figure 5), CD20, CD10 and CD138. Ki 67 proliferation index was of $98 \%$. Based on these studies, final histopathological and IHC diagnosis was unclassified B-cell lymphoma with intermediate features between DLBCL and cHL (WHO). A bone marrow biopsy was performed without evidence of atypical cells.

Patient was started on a new scheme of HAART based on tenofovir, raltegravir, darunavir plus ritonavir and was derived to an oncohematology center for chemotherapy. By his own decision, the patient was not available for his infectious monitoring.

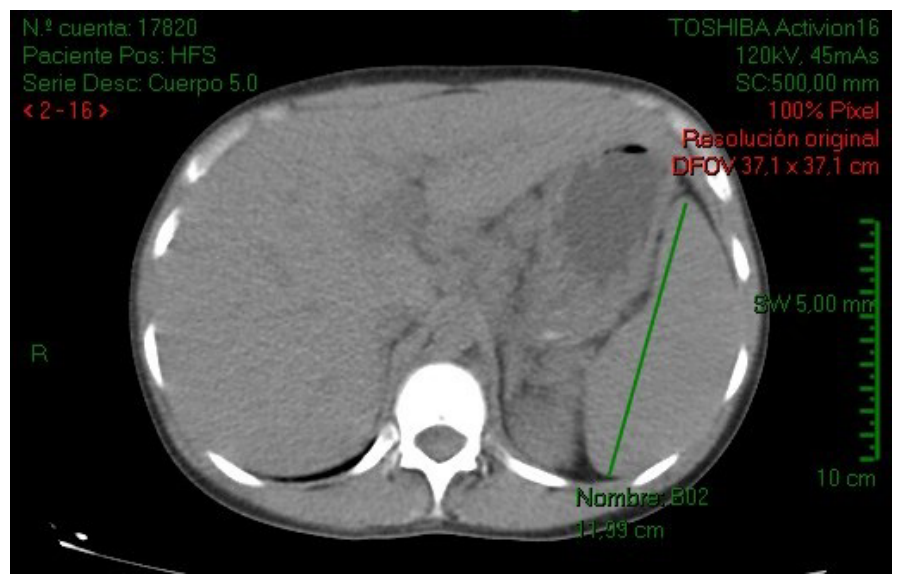

Figure 1. Abdominal CT scan showing hepatosplenomegaly

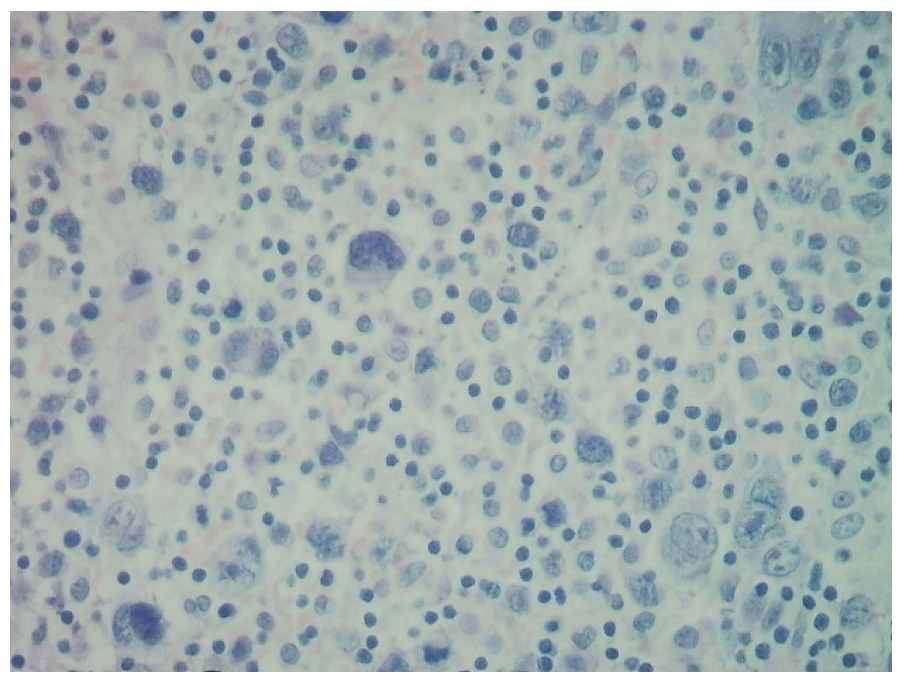

Figure 2. A dense cellular infiltrate with Reed Sternberg-like cells in a monomorphic background with eosinophils

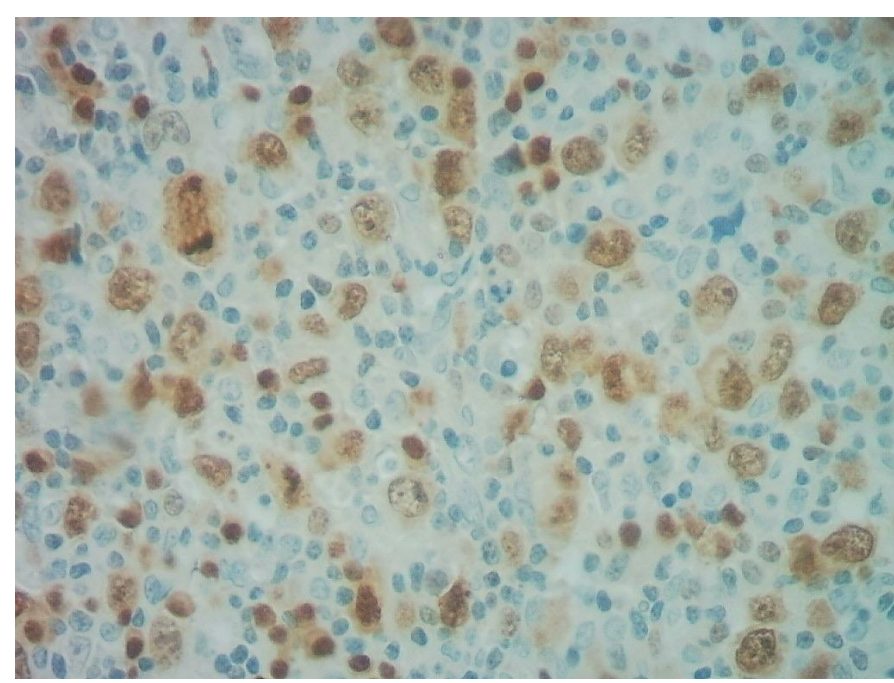

Figure 3. PAX5 positive expression in Reed-Sternberg-like cells

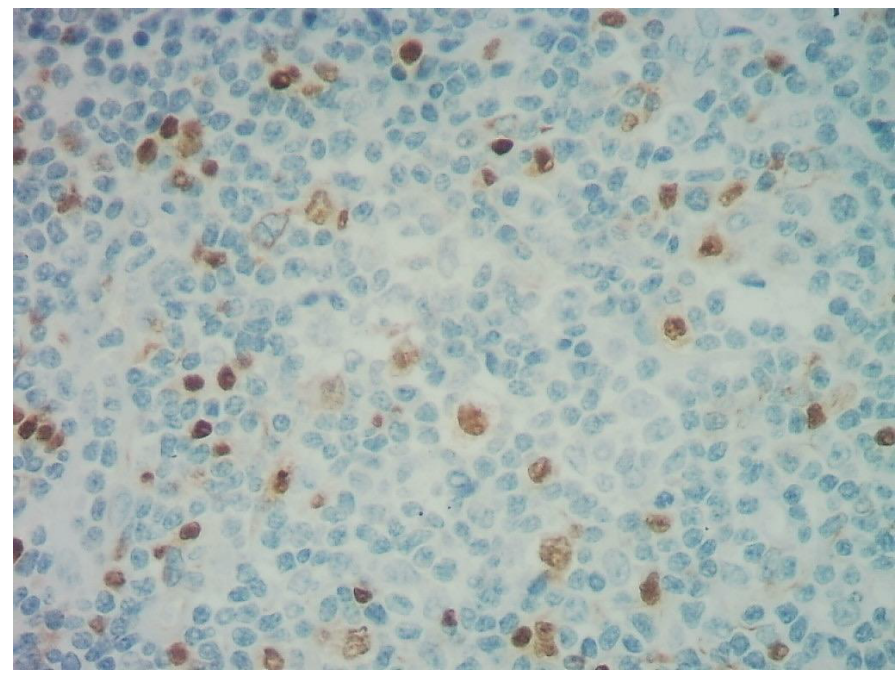

Figure 4. MUM-1 co-expression in Reed Sternberg-like cells

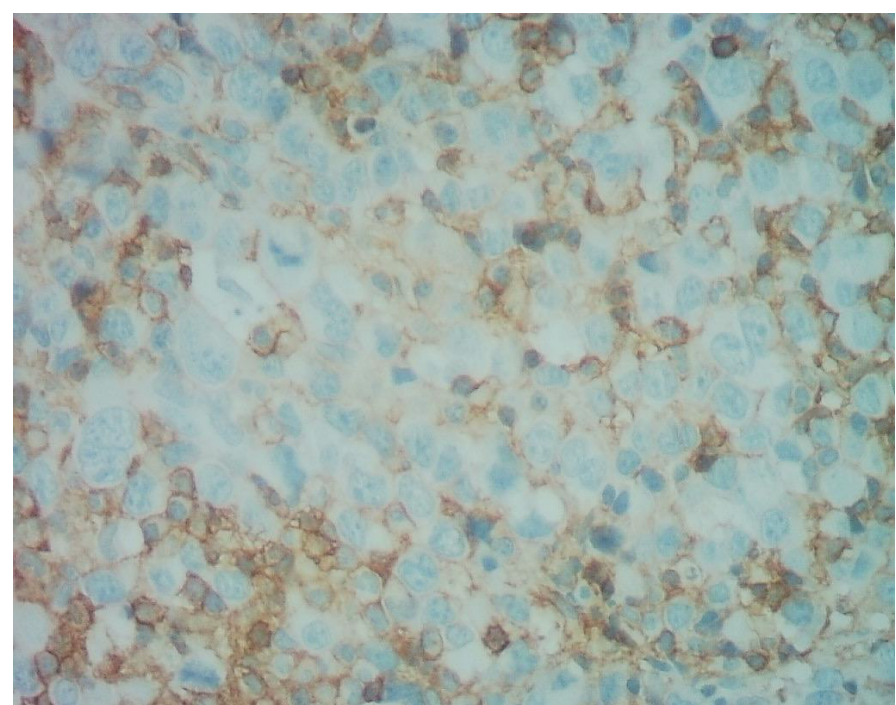

Figure 5. CD45 negative in atypical lymphoid cells 
Valerga M (2020) Unclassifiable B-cell lymphoma with features intermediate between diffuse large B-cell lymphoma and classical Hodgkin lymphoma in an AIDS patient. Report of a case and literature review

\section{Discussion}

Gene expression studies demonstrated differences between primary mediastinal DLBCL and classical HL and between primary mediastinal DLBCL and DLBCL $[8,9]$. There are few publications about the clinicopathological characteristics of unclassified DLBCL with intermediate features between DLBCL and classical HL in AIDS patients. Gualco G, et al. [10] analyzed 10 cases of unclassified lymphomas with features intermediate between DLBCL and classical HL. The authors remark the male preponderance ( 9 of 10 cases) with a median of age of 37 years and a marked frequency of clinical presentation as mediastinal masses (8 of 10 patients). Only one patient presented with an axillary lymph node enlargement and other with a retroperitoneal lymphadenopathy conglomerate [10]. In the case that we describe, the patient present cervical, mediastinal and retroperitoneal adenomegaly with homogeneous hepatosplenomegaly, indicating a high stage neoplasic disease.

In the serie of Gualco, et al. [10], the authors showed a high expression of CD79a and PAX5 indicative of B-cell immunophenotype with a low expression of BOB1. The expression of BOB1 is typical of B-cell lymphoma and can also be seen in nodular lymphocyte predominant HL and is absent in classical HL. Reed-Sternberg cells rarely express $\mathrm{BOB} 1$ [11]. In this series, all cases expressed at least two markers associated with B-cell phenotype and a good response to combination chemotherapy used for NHL [10]. Our patient presents the immunophenotypic characteristic of B-cell antigens, with the majority of cells expressing BCL2, PAX-5, OCT-2, BOB-1 and CD79a, with co-expression of CD30 and MUM-1. Also, some cells co-express CD23 and CD43. Ki 67 proliferation index was of 98\%. The unclassifiable B-cell lymphoma with features of classical HL is included in the WHO classification of lymphoproliferative disorders [5]. In other series, Sissolak G, et al. [12] evaluated retrospectively 35 patients with diagnosis of HIV-associated BL. Twenty four were typical $\mathrm{BL}$ and eleven had diagnosis of unclassified lymphoma with features of BL and DLBCL (BL/DLBCL, type-2). Patients with BL/DLBCL were significantly older, had less frequency of bone marrow infiltration and had lower levels of serum lactate dehydrogenase (LDH), compared with those with BL. A high percentage of patients presented advanced lymphoproliferative disease and $25 \%$ had central nervous system (CNS) involvement at the time of diagnosis. The authors used intensive regimens of chemotherapy based on hyper-CVAD with prophylactic intrathecal chemotherapy (Stanford regimen) and, in a less percentage, CHOP-like regimens. Thirty one patients received concomitant HAART. In this series, two years overall survival was just from $38 \%$, without differences between histopathological subtypes [12].
Further studies are necessary to clarify the histopathological characteristics and therapeutic options for this kind of patients with borderline aggressive lymphomas.

In conclusion, unclassified lymphoma is a severe complication of advanced HIV/AIDS disease. Early diagnosis followed by intensive chemotherapy plus HAART are necessary to improve the poor prognosis and the survival of these patients.

\section{References}

1. Kaplan LD, Abrams DI, Feigal E (1989) AIDS-associated non-Hodgkin's lymphoma in San Francisco. JAMA 261: 719-724. [Crossref]

2. Vaccher E, Spina M, di Gennaro G (2001) Concomitant CHOP chemotherapy and highly active antiretroviral therapy in patients with HIV-related non-Hodgkin's lymphoma. Cancer 91: 155-163.

3. Carbone A, Gloghini A, Aiello A (2010) B-cell lymphomas with features intermediate between distinct pathologic entities. From pathogenesis to pathology. Hum Pathol 41: 621-631.

4. Sabattini E, Bacci F, Sagramoso C (2010) WHO classification of tumours of haematopoietic and lymphoid tissues in 2008: an overview. Pathologica 102: 83-87. [Crossref]

5. Swerdlow SH, Campo E, Harris NL (2008) World Health Organization Classification of Tumours. Pathology and Genetics of Tumours of Haematopoietic and Lymphoid Tissues 3: 490.

6. Hummel M, Marafioti T, Stein H (1999) Clonality of Reed-Sternberg cells in Hodgkin's disease. $N$ Engl J Med 340: 394-395.

7. Kim J, Hahn J, Kim G, Yang W (2005) Three cases of diffuse large B-cell lymphoma presenting as primary splenic lymphoma. Yonsei Med J 46: 703-709. [Crossref]

8. Rosenwald A, Staudt LM (2003) Gene expression profiling of diffuse large B-cell lymphoma. Leuk Lymphoma 44(Suppl 3): S41-S47. [Crossref]

9. Rosenwald A, Wright G, Leroy K (2003) Molecular diagnosis of primary mediastinal B cell lymphoma identifies a clinically favorable subgroup of diffuse large B cell lymphoma related to Hodgkin lymphoma. J Exp Med 198: 851-862. [Crossref]

10. Gualco G, Natkunam Y, Bacchi CE (2012) The spectrum of B-cell lymphoma unclassifiable, with features intermediate between diffuse large B-cell lymphoma and classical Hodgkin lymphoma: a description of 10 cases. Modern Pathol 25: 661-674. [Crossref]

11. Minami J, Dobashi N, Asai O (2010) Two cases of mediastinal gray zone lymphoma. $J$ Clin Exp Hematop 50: 143-149. [Crossref]

12. Sissolak G, Seftel M, Uldrick TS, Esterhuizen TM, Mohamed N, et al. (2017) Burkitt's lymphoma and B-cell lymphoma unclassifiable with features intermediate between diffuse large B-cell lymphoma and Burkitt's lymphoma in patients with HIV: Outcomes in a South African Public Hospital. J Global Oncol 3: 218. [Crossref]

Copyright: (C2020 Valerga M. This is an open-access article distributed under the terms of the Creative Commons Attribution License, which permits unrestricted use, distribution, and reproduction in any medium, provided the original author and source are credited. 